\title{
Cancer Therapy Evaluation Program
}

National Cancer Institute

\section{Source}

National Cancer Institute. Cancer Therapy Evaluation Program. NCI Thesaurus. Code C15794.

The program within the Division of Cancer Treatment and Diagnosis (DCTD) of the $\mathrm{NCl}$ which plans, assesses, and coordinates all aspects of clinical trials including extramural clinical research programs, internal resources, treatment methods and effectiveness, and compilation and exchange of data as it pertains to the development and evaluation of anticancer agents. 